\title{
Dose standards for safe and secure breast cancer screening
}

\section{- Establishment of X-ray dose standards for mammography and construction of a calibration service system -}

\author{
Takahiro TANAKA*, Tadahiro KUROSAWA and Norio SAITO
}

[Translation from Synthesiology, Vol.5, No.4, p.222-233 (2012)]

\begin{abstract}
Breast X-ray screening (mammography) was introduced in 2000 to detect breast cancer at an early stage. Since then, the number of examinees has increased steadily. The X-ray dose must be optimized to obtain high-quality images that ensure diagnosis reliability without compromising safety. Mammography utilizes low-energy X-rays with a special energy spectrum for breast cancer screening. This $\mathrm{X}$-ray energy spectrum is significantly different from the reference X-ray spectrum used to calibrate dosimeters. Members of industry and academia were concerned about the reliability of dose evaluation for mammography quality control. Thus, the National Metrology Institute of Japan/National Institute of Advanced Industrial Science and Technology (NMIJ/AIST) has established an X-ray dose standard based on the X-ray energy spectrum for mammography and has disseminated this standard to industry. NMIJ has succeeded in rapidly establishing this standard by making the utmost use of existing research equipment and technology, and by utilizing the standard in the existing mammography quality control system. Moreover, it created a research and development (R\&D) scenario beforehand considering both domestic and international situations regarding mammography dosimetry that has enabled rapid and extensive dissemination of the standard with international consistency.
\end{abstract}

Keywords : Mammography, dosimetry standard, calibration, dosimeter, reliability

\section{Introduction}

Owing to the recent increase in mortality due to breast cancer, mammography was introduced in Japan from 2000 to ensure early detection of breast cancer. After its introduction, the number of mammography examinees has increased, and this number surpassed 2.5 million people in fiscal year (FY) 2009. To effectively diagnose breast cancer, high quality diagnostic images and excellent interpretation techniques (e.g., the ability to discern lesions on diagnostic images) are required. However, to ensure patient safety, mammography must be performed with a minimum yet appropriate X-ray dose. The maintenance and improvement of mammography quality control are mainly conducted by academic societies and industries. Further improvement in the reliability of mammography X-ray dose assessment was demanded by the societies and industries involved because of the large discrepancy between the radiation quality (energy spectrum) for mammography and the calibration reference field, and this difference might affect the sensitivity of the dosimeters. Therefore, to manage this issue, the National Institute of Advanced Industrial Science and Technology (AIST) set out to develop and disseminate the national standard for X-ray dose based on mammography X-rays.

Measurement standards become meaningful only when they are used widely in society, and it is therefore important to meet the social demand for such standards in a timely manner. The typical period required for the development of a radiation dose standard is approximately $3-5$ years. Here, by fully utilizing existing research facilities and technologies, the period from the commencement of development to dissemination was reduced to approximately 1.5 years. Moreover, a widely available calibration service system was established rapidly by utilizing the standard in the current quality control system for mammography. In this paper, we describe the scenario established by AIST for developing the mammography X-ray standard and improving the reliability of X-ray dose evaluation in mammography quality control.

\section{Background of the research and development}

\subsection{Expansion of mammography in society}

The age-adjusted incidence rate $\mathrm{T}^{\mathrm{Term} 1}$ and the age-adjusted mortality rate ${ }^{\mathrm{Term} 2}$ for breast cancer has been increasing steadily in recent years. ${ }^{[1]}$ Because the prognosis of breast cancer is good when treated early, early detection leads to a decrease in mortality. The rapid increase in the number of breast cancer patients was seen much earlier in Europe and in the US than in Japan. Since mammography was introduced as a screening methodology for the early detection of breast cancer, the mortality rate of breast cancer has decreased. ${ }^{[2]}$

In Japan, in addition to visual inspection and palpation, mammography was introduced for breast cancer screening beginning in 2000. The Ministry of Health and

National Metrology Institute of Japan, AIST Tsukuba Central 2, 1-1-1 Umezono, Tsukuba 305-8568, Japan

* E-mail : takahiro-tanaka@aist.go.jp

Original manuscript received January 31, 2012, Revisions received August 7, 2012, Accepted August 15, 2012 
Welfare (currently the Ministry of Health, Labour and Welfare $)^{[3]}$ recommended that patients aged 50 and older undergo screening ( $4^{\text {th }}$ Elderly Health Care Project). The recommendation was revised in $2004,{ }^{[4]}$ and the age range was expanded to 40 years and older $\left(5^{\text {th }}\right.$ Elderly Health Care Project). Enforcement of the Cancer Control Act began in 2007, and since March 2008, cancer screening was included in the health promotion project (based on the Health Promotion Act). With the expansion of the age range recommended for screening, the number of examinees has increased since the introduction of mammography in 2000, and this number surpassed 2.5 million in FY 2009. ${ }^{[5]}$

\subsection{Characteristics of mammography X-rays}

In all diagnostic procedures involving $\mathrm{X}$-rays, including mammography, an X-ray tube is used as the X-ray source. This tube is a device that generates X-rays by accelerating thermal electrons released from the filament to a metal plate (target) using high voltage (several $\mathrm{kV}$ to several hundred $\mathrm{kV}$ ). The high voltage applied between the filament and target is called the tube voltage. These X-rays may include both characteristic and Bremsstrahlung X-rays depending on the combination of the target material and the tube voltage. The energy spectrum (radiation quality) is altered by passing the X-rays generated from the X-ray tube through a pure metal filter. The tube voltage and the material of the added filter and its thickness are adjusted to obtain X-rays with optimal quality for a given subject. Since the energy spectrum (radiation quality) is strongly depends on the target material and the filter, the expression of "target material / filter material" is commonly used. For example, if the target material is tungsten (W) and the filter is aluminum ( $\mathrm{Al}$ ), the energy spectrum (radiation quality) is expressed as W/Al.

Compared to general radiography such as that used for the chest, the X-rays used in mammography have 2 features: (1) they have lower energy and (2) their energy spectra include characteristic X-rays.

Regarding X-ray energy, while a tube voltage of approximately $80 \mathrm{kV}$ is used in general $\mathrm{X}$-ray radiography, $30 \mathrm{kV}$ is used for mammography. Because the difference in the linear attenuation coefficient ${ }^{\text {Term } 3}$ between glandular tissues and tumor tissue becomes larger as the X-ray energy decreases, low-energy X-rays are necessary to obtain high contrast images. However, X-ray absorption by the skin increases as the X-ray energy decreases. Therefore, in mammography, an X-ray tube voltage of approximately 30 $\mathrm{kV}$ is used to maintain both dose and image quality. At a tube voltage of approximately $30-80 \mathrm{kV}$, the absorption per unit length of matter, including air as well as the material of the dosimeter, increases as the X-ray energy decreases, which is a factor that prevents highly precise dosimetry.

Another characteristic of mammography X-rays is their energy spectrum. Tungsten (W) is used as the target material of the X-ray tube in general X-ray radiography, whereas molybdenum (Mo) is mainly used in mammography. In mammography, the tube voltage settings between 25 and 35 $\mathrm{kV}$ are typically used, depending on breast tissue thickness and composition. This is because the tube voltage is above the ionizing energy of the Mo K-shell and characteristic $\mathrm{X}$-rays are emitted in abundance. For mammography, a Mo target X-ray tube and a Mo additional filter are typically used. As a result, the energy spectrum contains an abundance of characteristic X-rays as shown in Figure 1. Using the Mo additional filter, all except the characteristic $\mathrm{X}$-rays are filtered out, and the energy spectrum becomes monochromatic. This combination is used because X-rays with lower energy than the Mo characteristic X-rays lead to relatively strong radiation exposure of the skin, whereas high-energy X-rays decrease the image contrast.

As described above, mammography is characterized by the use of low-energy X-rays that contain an abundance of characteristic X-rays.

\subsection{Dosimetry in mammography}

As an aid to the optimization of the radiation protection of the patient, dosimetric quantities need to be measured for radiography systems. In general X-ray radiography such as chest radiography, for example, the absorbed dose to the skin is used in dose evaluation.. However, in mammography, dose evaluation is done using a special dose called the average glandular dose (AGD) for the following reasons:

- only the breasts are exposed to X-rays,

- the glandular tissues are assumed to be most vulnerable to radiation in the breast, and

- the dose changes rapidly within the breast because the absorption per unit length is large, which is because of the low energy of the X-rays used.

The AGD is calculated as the X-ray dose absorbed by all glandular tissues within the breast divided by the total mass

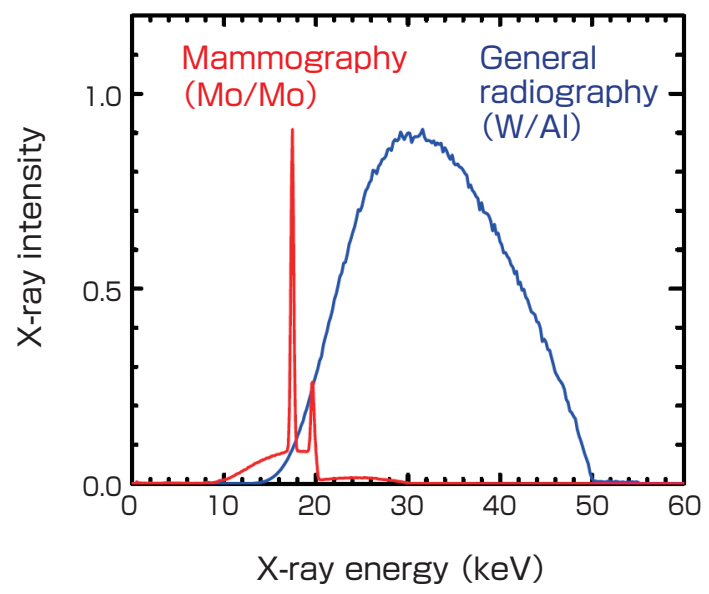

Fig. 1 Examples of the X-ray spectra for mammography (Mo/Mo) and general radiography (W/AI) 
of the glandular tissues (the absorbed dose per unit mass of glandular tissue). The International System (SI) of units for AGD is $\mathrm{J} / \mathrm{kg}$, and this unit is designated as grey (Gy). The AGD is used as a representative of the absorbed dose to glandular tissues, which decreases as X-rays penetrate deeper into the breast (direction from the X-ray source to the receiving device, see Fig. 2). However, the AGD may change according to the amount (percentage) and distribution of the glandular tissues within the breast or the compressed breast thickness. Therefore, in mammography quality control, the standard breast is a breast with a thickness of $45 \mathrm{~mm}(42$ $\mathrm{mm}$ in some cases) with uniform distribution of adipose and glandular tissues by weight. The AGD is evaluated using this standard definition of a breast. ${ }^{[6] 7]}$ To optimize (minimize) the AGD, reference levels (or minimum dose targets) have been set by related academic societies.

The AGD for an individual human body is extremely difficult to evaluate, and it is evaluated using a phantom, which is a material that simulates the standard breast as recommended by academic societies. Figure 2 shows an evaluation of AGD using a mammography machine.

The dosimeter is placed so that its reference plane matches the surface of the phantom, and the dose of X-rays irradiated onto the phantom surface (X-ray source side) is measured. Because the dose measured by the dosimeter in this case is expressed by the unit "air kerma" ${ }^{\text {Term } 4}$, it is necessary to convert from air kerma to AGD. This conversion factor can be determined using the Monte Carlo calculation, and the tables for corresponding X-ray qualities are given in quality control manuals and other references. ${ }^{[6][7]}$ Therefore, to evaluate the AGD, an evaluation of the radiation quality of X-rays from the mammography machine is necessary.

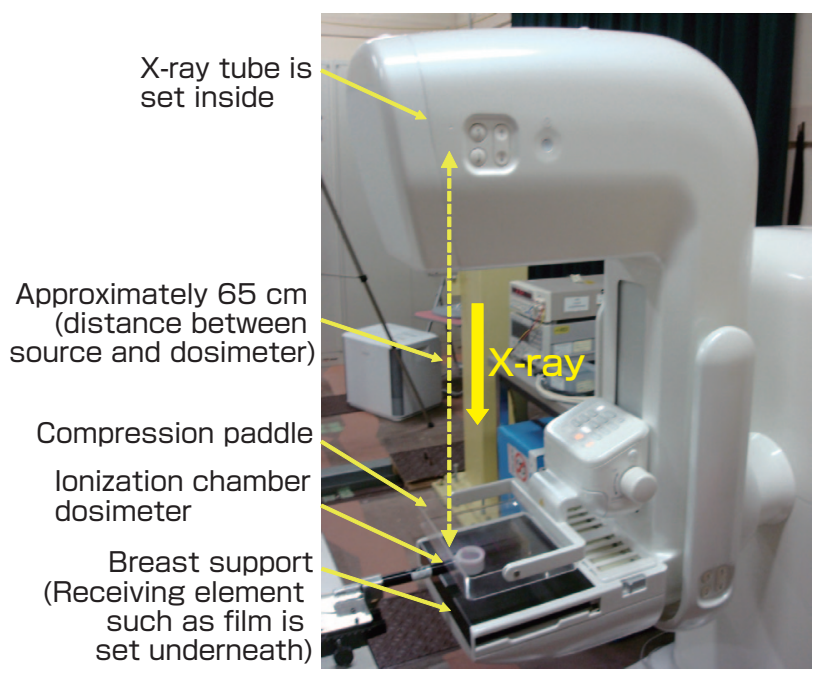

Fig. 2 Example of the installation of a dosimeter for the dose evaluation of a mammography machine

The reference plane of the dosimeter is lifted from the breast support because of the thickness of the phantom. In the photograph, only the ionization chamber dosimeter is mounted.
However, the measurement of an energy spectrum in actual medical practice is not realistic considering the time and cost. Therefore, the mammography radiation quality is expressed as the thickness of the material (aluminum is used in mammography) required to reduce the amount of air kerma by half. This thickness is called the half-value layer. As described above, it is necessary for evaluation of the AGD to measure the dose (air kerma) and quality (half-value layer) using a dosimeter.

\subsection{Dosimeters for mammography $X$-rays}

There are many types of dosimeters with different measurement principles. In the medical practice of mammography, 2 types of dosimeters are widely used: the ionization chamber type and the semiconductor type.

The ionization chamber dosimeter measures the amount of ionization (ion-electron pair) generated by the interaction between X-rays and air. In mammography, X-rays with low energy (or those strongly attenuated by matter) are used. Therefore, the entrance window in an ionization chamber used for mammography X-rays is composed of a thin film (mainly metal vapor-deposited resin) that allows for high transmittance of X-rays. Because an ionization chamber can measure the X-ray dose close to the definition of air kerma, it is used as a reference dosimeter. However, because the volume of air changes according to environmental conditions such as temperature and pressure, correction for environmental conditions is necessary. Because the X-ray entrance window is composed of a thin film, care must be taken in handling the device. Additionally, because the thin film absorbs X-rays, the sensitivity of the ionization chamber is likely to change depending on the X-ray energy, which may create problems for the measurement of low-energy X-rays.

Silicon is mainly used in semiconductor dosimeters, and the depletion layer formed by the P-N junction (application of reverse bias voltage) is used as the radiation sensitive layer. In the PIN-type semiconductor dosimeters, the intrinsic semiconductor layer (i layer) acts as the radiation sensitive layer. While the carrier of the current is the electron-ion pair in the ionization chamber dosimeter, the current is carried by the electron-hole pair in the semiconductor dosimeter. Therefore, the semiconductor dosimeter is referred to as a solid ionization chamber. Compared to the ionization chamber dosimeter, the semiconductor dosimeter is sturdy and convenient to use with no correction required for temperature and pressure, and it is therefore used frequently for dose evaluation in actual medical practice. However, because the surface $\mathrm{SiO}_{2}$ layer and the non-sensitive layer absorb a large amount of X-rays, the sensitivity is greatly dependent on X-ray energy in the low-energy region such as mammography X-rays. 
Both the ionization chamber and semiconductor dosimeters have been developed as dosimeters for mammography X-rays (or low-energy X-rays); however, the energy dependence of the sensitivity (hereinafter energy dependence) cannot be avoided because of the physical structure of the dosimeters. Therefore, for the measurement of the X-ray energy region used in medical practice, academic societies recommend calibration of the dosimeters using a reference X-ray field with accurately determined characteristics.

\subsection{International trend}

Because the rapid increase in the number of patients with breast cancer was seen earlier in Europe and the US than in Japan, the construction of quality control systems started much earlier in Europe and the US. In the US, the American College of Radiology (ACR) established a quality standard for mammography in 1986 and began work on quality control. In 1992, the Mammography Quality Standard Act was established as a federal law, and mammography screening became legislated. ${ }^{[8]}$ Under the mammography screening law, all facilities conducting mammography screenings must receive certification from inspection institutes (ACR or state governments) accredited by the Food and Drug Administration (FDA) and are required to undergo medical inspection and receive clearance from the FDA. This law states that the dosimeter must be calibrated once every 2 years, and traceability to the national standard must be guaranteed. The National Institute of Standards and Technology (NIST), which is responsible for the American national standard, disseminates the mammography air kerma standard based on Mo/Mo radiation qualities. The Quality Control Manual ${ }^{[9]}$ published by ACR was adopted into a quality control manual for Japan.

The European Reference Organization for Quality Assured Breast Screening and Diagnostic Services (EUREF) played a central role in creating the guideline for mammography quality control in Europe. ${ }^{[10]}$ This guideline states that the dose must be evaluated once every 6 months. European countries conduct quality control using individual methods that are based on this guideline. The air kerma standard based on Mo/Mo qualities was disseminated mainly in Germany and the UK. However, several European institutes calibrate mammography dosimeters using the W/A1 qualities, and there were concerns that differences in radiation quality may affect the calibration results. Therefore, institutes belonging to the European Collaboration in Measurement Standards (EUROMET, currently known as the European Association of National Metrology Institutes; EURAMET) performed an international comparison for the calibration of mammography dosimeters.

In this international comparison, several ionization chamber and semiconductor dosimeters were circulated and calibrated with the radiation qualities (either Mo/Mo or W/A1) used by the institutes, and the calibration results were compared. The results indicated that for the dosimeters with a small energy dependence, such as the ionization chambers for mammography (soft X-rays), the effect of radiation quality on the calibration result was not a major issue in practice. However, dosimeters with a large energy dependence, such as the semiconductor dosimeters, should be calibrated under radiation quality conditions similar to that of mammography $\mathrm{X}$-rays (such as Mo/Mo). ${ }^{[1]}$

With this background, the Consultative Committee for Ionizing Radiation in the International Bureau of Weights and Measures (Bureau International des Poids et Mesures; BIPM) made a resolution that the BIPM establish the air kerma standard for mammography based on the Mo/Mo radiation qualities. This means that the dosimeters should be calibrated using the air kerma standard for mammography radiation qualities. BIPM prepared the reference field for mammography X-rays based on Mo/Mo radiation qualities, and an international comparison with BIPM began in 2009. ${ }^{[12]}$

\subsection{Societal need for the mammography X-ray air kerma standard}

With low-energy X-rays such as those used in mammography, the energy dependence of the dosimeter (both ionization chamber and semiconductor types) is large, and academic societies recommended that the calibration be performed at an energy close to that of the X-rays used in medical practice. Conventionally, the dose standard of X-rays in the mammography energy region was disseminated with W/ A1 radiation qualities. Figure 3 illustrates an example of the energy dependence of the ionization chambers (range of

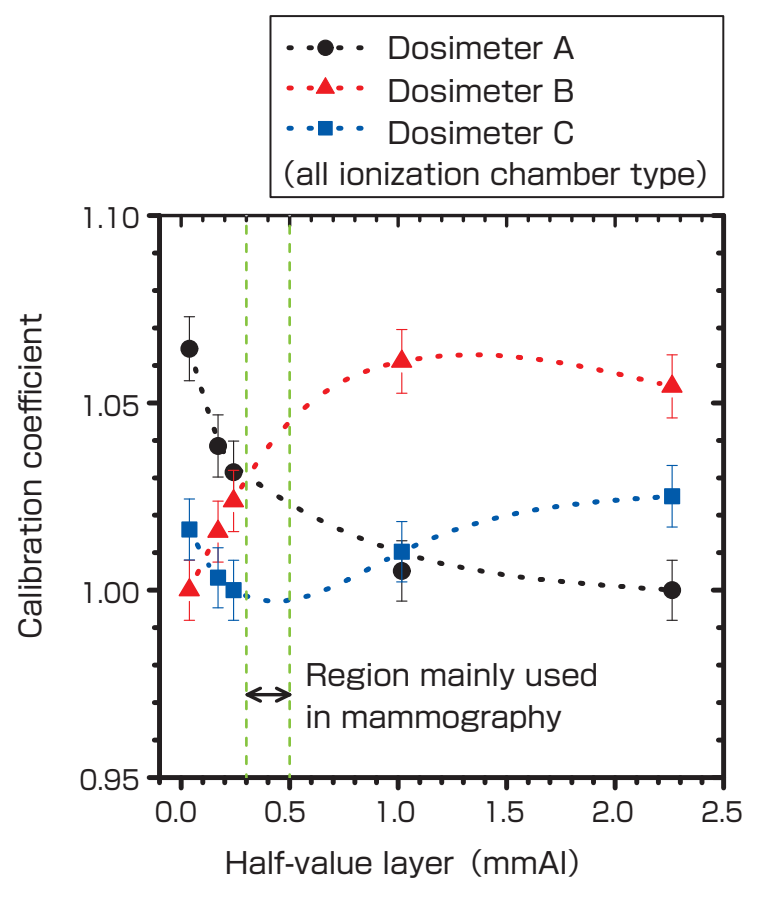

Fig. 3 Example of the energy dependence of the ionization chamber 
the half-value layer for AIST's soft X-ray standard of W/A1 radiation qualities).

In the X-ray energy range used in mammography, the sensitivity (calibration coefficient) of ionization chambers with a relatively small energy dependence changes with X-ray energies more than their uncertainties $(95 \%$ confidence interval) as shown by the vertical bar for each data point in Figure 3. The change in sensitivity differs according to the type of dosimeter, i.e, the calibration coefficients of the dosimeter A decrease with the HVL whereas those of dosimeter B increase. This change is due to differences in the material and thickness of the X-ray entrance window of the dosimeter as well as the internal structure of the dosimeter. The semiconductor dosimeters, which have a larger energy dependence than the ionizing chambers, are used more often in medical practice; thus, Japanese industries and academic societies were concerned about the effect of the radiation quality and energy dependence of the dosimeters on the calibration coefficient. To manage this problem, AIST developed and disseminated a dose standard based on the radiation qualities of mammography X-rays.

\section{Scenario for improving the reliability of mammography $\mathrm{X}$-ray dose evaluation}

To improve the reliability of mammography dose evaluation in medical practice, it is necessary to (1) establish a dose standard based on the radiation qualities of mammography X-rays used in practice and (2) create a calibration service system to disseminate the dose standard to society. Figure 4 shows a scenario for improving the reliability of mammography dose evaluation.
To quickly respond to social demands, time required for standard development was shortened by utilizing the existing primary standard without developing a new primary standard optimized for mammography X-rays. To reduce the uncertainty of dose evaluation in medical practice, to which the standard was mainly disseminated, an X-ray field close to that of an actual mammography machine was developed. Additionally, to confirm the international equivalence of the standard, an X-ray reference field was developed at the same time in compliance with the International Electrotechnical Commission (IEC) standard. In addition to participating in the international key comparison, the calibration capability of the developed dose standard was verified by comparing the dose measurements of X-rays from an actual mammography machine with several dosimeters calibrated using the standard, and the results were evaluated.

Because mammography quality control (including dose evaluation) was already being performed in many medical practices, rapid and widespread dissemination of the standard was achieved by evaluating the glass dosimeter that was already being used in this quality control. Therefore, AIST developed a method for calibrating the glass dosimeters.

In the conventional traceability scheme of the dose standards, the standards were disseminated through networks of secondary calibration laboratories by means of calibrations. In this case, it would not be useful if the conventional W/A1 radiation quality owned by the calibration laboratories was used as the reference (X-ray) field. However, it costs several tens of millions of yen to develop a reference field for the radiation qualities of mammography X-rays. Therefore, AIST developed a system in which the AIST's irradiation facility could be used for the calibration/testing service to ensure

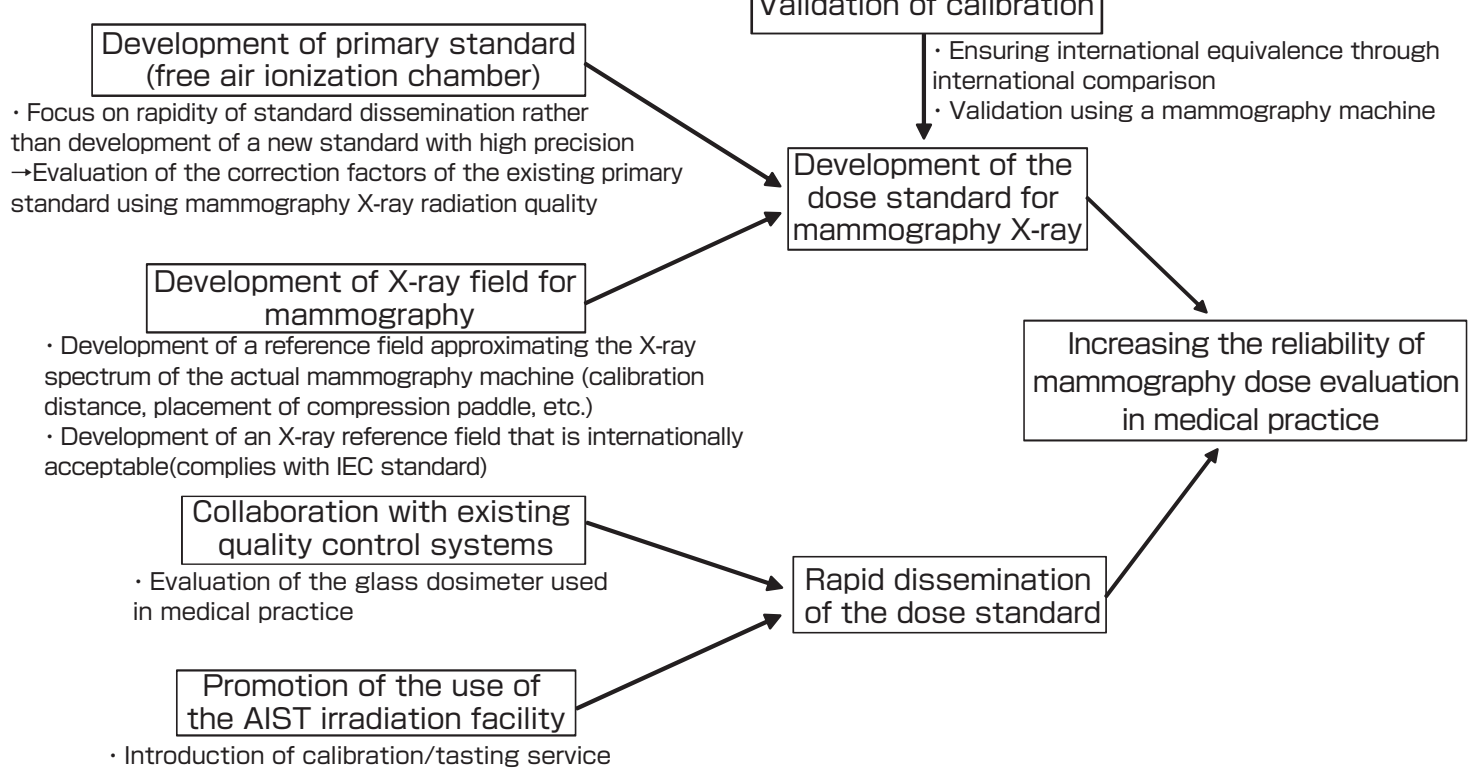

Fig. 4 Scenario for improving the reliability of mammography X-ray dose evaluation 
smooth dissemination of the standard.

This scenario was created to ensure rapid and widespread dissemination of the internationally equivalent dose standard to medical practitioners and to improve the reliability of dose evaluation.

\section{Development of the national primary standard}

To develop a primary standard for mammography X-ray dose, a primary standard instrument for absolute dose measurement (measurement according to the definition of a unit) and a reference X-ray field with the same radiation quality as mammography X-rays must be developed. The development of a primary standard instrument and a mammography X-ray reference field will be described in these sections.

\subsection{Development of the primary standard instrument} In the case of soft X-rays (here, a tube voltage of $50 \mathrm{kV}$ or less), including those used in mammography, the dose standard (in Gy [or $\mathrm{C} / \mathrm{kg}]$ ) has already been disseminated for the air kerma ${ }^{\text {Term } 4}$ (or exposure ${ }^{\text {Term } 5}$ ) that has been physically defined.

The free air ionization chamber, which is capable of absolute measurement of the radiation dose, is used worldwide as the primary standard instrument. Currently, this instrument is used as the primary standard instrument for the measurement of soft X-ray doses at AIST (Fig. 5).

In the free air ionization chamber, the ion charge generated within the ionization volume is measured, and the radiation dose (or air kerma) is evaluated. When the mass of air within the ionization volume is set as $m$, the exposure rate $\dot{X}\left(\mathrm{C} \mathrm{kg}^{-1} \mathrm{~s}^{-1}\right)$

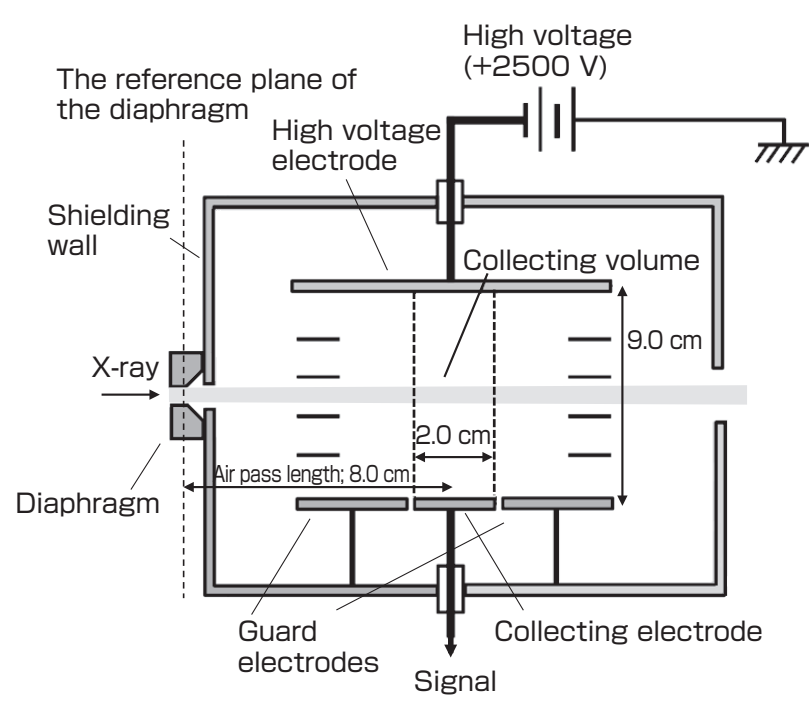

Fig. 5 Schematic diagram of the primary standard (free air ionization chamber) is obtained using equation (1).

$$
\dot{X}=\frac{I}{m} \prod_{i=1}^{10} k_{i} \quad \cdots(1)
$$

In this equation, $I$ is the ionization current measured by the free air ionization chamber and $\Pi k_{i}$ is the product of the correction factors. The correction factors are used to correct for the deviation between the actual experimental condition and the ideal condition defined for the dose, and there are a total of 10 different correction factors. For mammography X-rays, the largest correction factor (approximately $1.5-2 \%$ ) is the correction for the attenuation of X-ray along the air path between the reference plane and center of the collective electrode. There is a minor correction (approximately $0.5 \%$ ) for scattering radiation, and the rest of the correction factors are fairly small (less than $0.1 \%$ ). The correction factors that are difficult to experimentally evaluate, such as the correction for scattering radiation, are evaluated using the Monte Carlo simulation.

Figure 6 shows a photograph of the present mammography dose standard equipment. To develop the national standard for mammography, the correction factors of the free air ionization chamber (the primary standard instrument for soft X-ray dose) were evaluated for the radiation quality of mammography X-rays without developing a new primary standard instrument (free air ionization chamber) specific for mammography X-rays. This significantly shortened the time required for the development of a standard and allowed for a quick response to the social demand for dissemination of the standard. As shown in Figure 6, the free air ionization chamber is installed on the XY stage and can be shared with the dose standard for soft X-rays (W/A1 radiation quality).

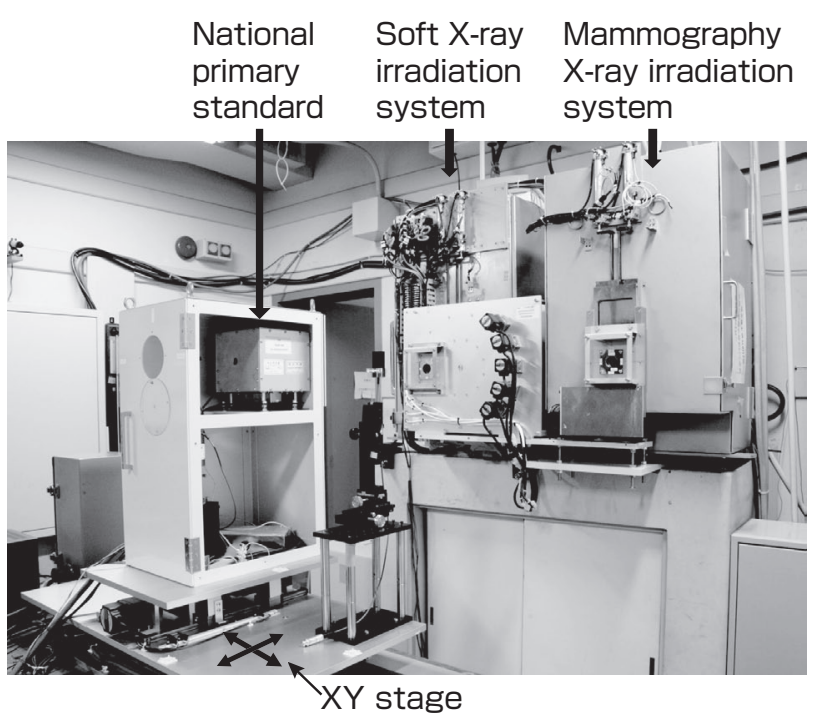

Fig. 6 Apparatus used for developing the mammography $X$-ray dose standard 


\subsection{Development of the mammography X-ray reference field}

A reference field for the radiation quality of mammography $\mathrm{X}$-rays was developed starting mainly from the dominant radiation qualities used in practice. As mentioned earlier, the radiation quality is determined by the target material of the $\mathrm{X}$-ray tube, the tube voltage, and the material and thickness of the additional filter. We ensured that we were using a radiation quality that could be used overseas as well as in Japan, as exemplified by the International Organization for Standardization (ISO) and IEC standards (for mammography, IEC 61267). ${ }^{[13]}$ Such a radiation quality is necessary for international key comparisons that are conducted to confirm the equivalence of the dose standard around the world. However, a radiation standard other than that of the IEC is used in the Japanese quality control manuals, and compliance with the Japanese standard would lead to a smooth dissemination of the standard. Consideration of both overseas and domestic situations when establishing a standard is the first step toward disseminating a dose standard that is internationally equivalent.

Because the linear absorption coefficient for air that is used in mammography is larger than that used in other diagnostic $\mathrm{X}$-ray modalities, the radiation quality changes significantly because of the calibration distance (distance from the focus point of the X-ray tube to the reference plane of the free air ionization chamber); therefore, it is important to set the calibration distance. In countries such as Germany and the US that have begun disseminating standards before Japan, the calibration distance is $1 \mathrm{~m}$. However, AIST set the calibration distance at $60 \mathrm{~cm}$ considering the irradiation distance of the mammography machine. Later, BIPM set the calibration distance at $60 \mathrm{~cm}$.

A dose standard based on the radiation quality through the compression paddle was originally developed in Japan. In actual mammography screening, the breasts are irradiated with X-rays through the compression paddle. The lowenergy X-rays used in mammography tend to be absorbed by the compression paddle, and the radiation quality changes greatly. Thus, an original radiation quality was developed considering the quality of radiation exposure to the breasts. This radiation quality was required for the calibration of the dosimeter used in mammography quality control.

\section{Construction of the calibration service system for mammography}

The construction of a calibration service is essential for improving the reliability of dose evaluation in medical practice. Therefore, multiple calibration services were established with the cooperation of industries and academic societies.

\subsection{Performance test of the glass dosimeter used for mammography quality control}

Before mammography was utilized for breast cancer screening in Japan, mammography quality control was performed mainly by related academic societies. A mammography glass dosimeter was developed to easily evaluate the dose and the radiation quality necessary for mammography quality control.

The glass dosimeter is an integrated-type radiophotoluminescence ${ }^{\text {Term } 6}$ dosimeter, and silver-activated phosphate glass is used as the fluorescent glass element. Figure 7 shows a photograph of the mammography glass dosimeter.

The mammography glass dosimeter consists of a fluorescent glass element and aluminum filters with 4 different thicknesses covering the surface of the element. The thicknesses of the aluminum filters are $0.3,0.4,0.6$, and 1.0 $\mathrm{mm}$, and an attenuation curve can be obtained from one irradiation. The half-value layer and tube voltage can be calculated from this attenuation curve, and the air kerma can be calculated from the amount of fluorescence at the open window (without the Al filter) glass element. Thus, the AGD required for mammography quality control can be evaluated with only one irradiation. The performance of this glass dosimeter was evaluated using the reference field.

Our results confirmed that the half-value layer, tube voltage, and air kerma values are in good agreement with the values measured using the free air ionization chamber (within $2 \%$ uncertainty at the $95 \%$ confidence level). ${ }^{[14]}$

\subsection{Use of the irradiation facility at AIST}

A mammography X-ray source is necessary for calibration laboratories to perform calibration using the radiation quality of mammography X-rays. However, such an X-ray source costs a minimum of tens of millions of yen; therefore, the calibration service would not be viable as a business. To solve the issue, AIST initiated a calibration/testing service in which the calibration laboratory is allowed to bring the reference dosimeter (assumed to be traceable to the primary standard) and the dosimeter to be calibrated to the mammography

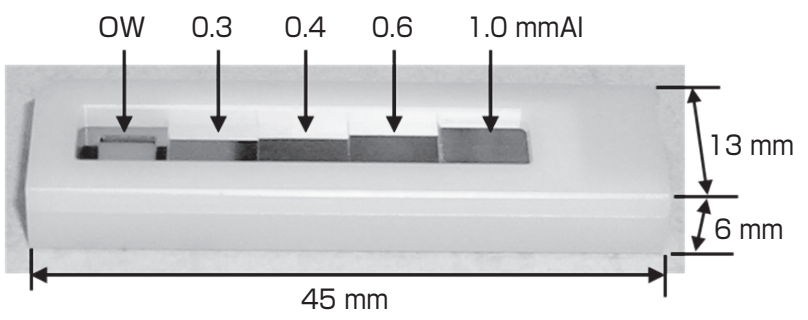

Fig. 7 Glass dosimeter developed for quality control of mammography (with cooperation from Chiyoda Technol Corporation) 
reference field at AIST to perform calibration. The fees for the calibration/testing service are calculated according to the number of days the irradiation facility is used. Therefore, the expenditure that must be borne by the calibration labs was reduced, and a smooth dissemination of the standard was expected.

\section{Validation of the calibration}

\subsection{Confirmation of the international equivalence of the national standard}

To confirm international equivalence, all standards must be compared with the standards of other countries. As mentioned earlier, BIPM developed the dose standard for mammography X-rays and has performed key comparisons ${ }^{\text {Term } 7}$ since 2009. AIST participated in the international key comparison in 2009. ${ }^{[15]}$

There are 2 methods for international comparison of a radiation dose standard. The first method is a direct comparison between the primary standards of the countries involved. For example, the primary standard instrument (free air ionization chamber) at AIST is taken to the reference field at BIPM, and the absolute values of the dose are compared. This method is limited to cases in which the primary standard instrument is transferrable. Another method is an indirect comparison, in which the transfer instruments are calibrated using the primary standard at the institutes involved and the calibration results (calibration coefficient) are compared.

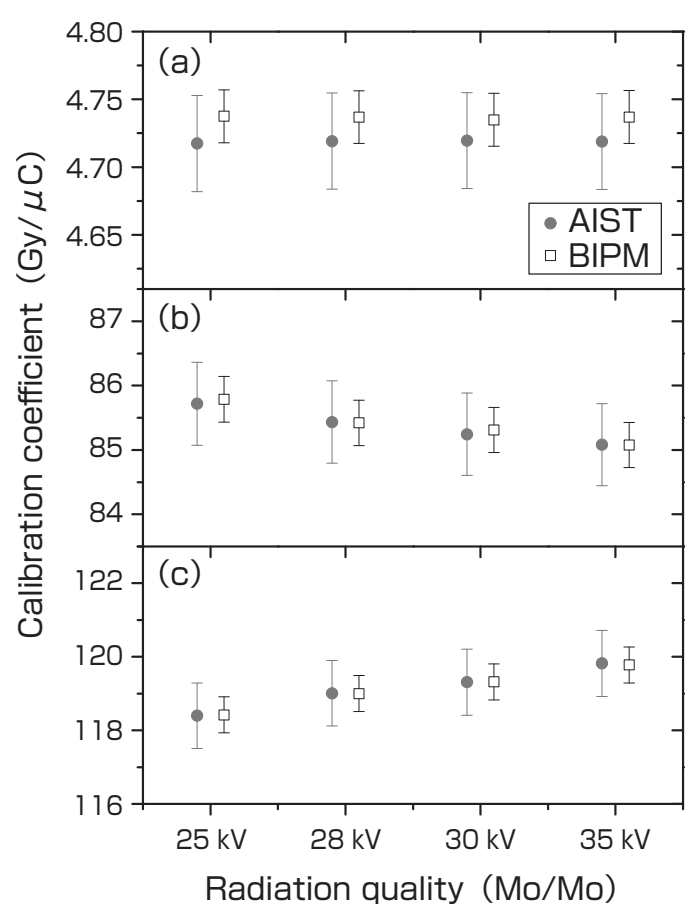

Fig. 8 Comparison between AIST and BIPM calibration coefficients of 3 types of dosimeters ( $a, b$, and $c)$

The energy characteristic of the dosimeter is (a) flat, (b) declining, and (c) increasing.
This method is useful in cases where the primary standard instrument cannot be transported easily because of its size.

The free air ionization chamber, which is the standard instrument used for measuring mammography X-rays at AIST, is also used to determine the conventional soft X-rays (W/Al) dose. This standard instrument was compared directly with the BIPM standard in 2004, and its equivalence was confirmed. ${ }^{[16]}$ Therefore, in this key comparison, the latter method of the indirect comparison was selected. Three ionization chamber dosimeters with different energy characteristics were selected for a detailed comparison. Figure 8 shows a comparison of the calibration results obtained for the 3 types of dosimeters measured at the 2 institutes.

As shown in Figure 8, the calibration coefficients of the dosimeters showed good agreement between institutes. The calibration uncertainty of BIPM (error bar in Fig. 8) was smaller than that of AIST for all 3 types of dosimeters because BIPM has a newly developed free air ionization chamber that has been optimized (i.e., the correction coefficient for equation (1) is small) for the radiation quality of mammography X-rays.

Figure 9 shows the results of the international key comparison for mammography dose standards. Germany (the PhysikalischTechnische Bundesanstalt, PTB), the US (NIST),

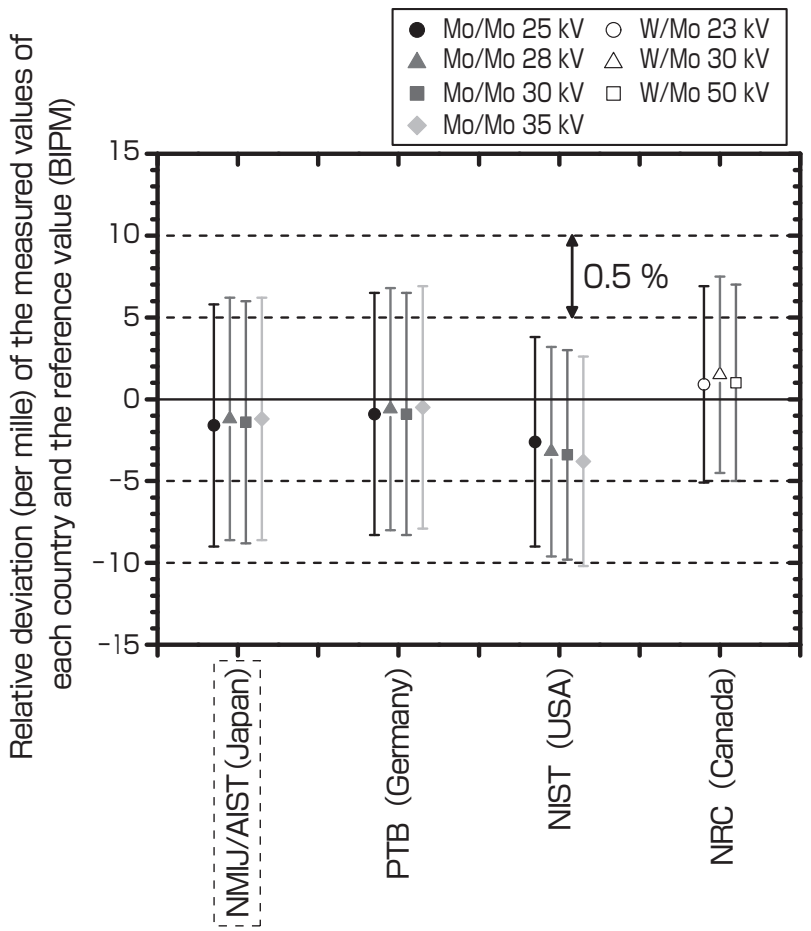

Fig. 9 International key comparison for the mammography dose standard ${ }^{[17]}$

The vertical axis shows the deviation (per mille) from the BIPM reference value. For each data point, the vertical bar shows the uncertainty at the $95 \%$ confidence level. 
and Canada (the National Research Council, NRC, radiation quality is slightly different) participated in this international key comparison, and equivalence was confirmed among the mammography dose standards at all institutes. ${ }^{[17]}$ It was also found that the uncertainty in the AIST dose standard was comparable to that in the dose standards of other countries.

\subsection{Validation using a mammography machine}

The X-rays produced by mammography machines used in medical practice are generated as a short pulse. However, the dose rate at the reference field is constant over time because stability is required. Additionally, in an actual mammography machine, the structure of the irradiation device is compact and it is difficult to put in the national primary standard, whereas there is spatial allowance in the standard field. The investigation of such differences between an actual mammography machine and the standard field will help improve the reliability of dosimetry in medical practice. Therefore, AIST conducted a comparison of the dose evaluation results between ionization chamber dosimeters (calibrated at AIST) and a glass dosimeter using a mammography machine employed in medical practice.

As a result, it was confirmed that the AGDs measured by the ionization chambers and glass dosimeter agreed within the uncertainties (Fig. 10). The dosimeters calibrated at the AIST's standard field were found to be reliable for dose evaluation of an actual mammography machine.

\section{Conclusions}

AIST has developed a dose standard for mammography

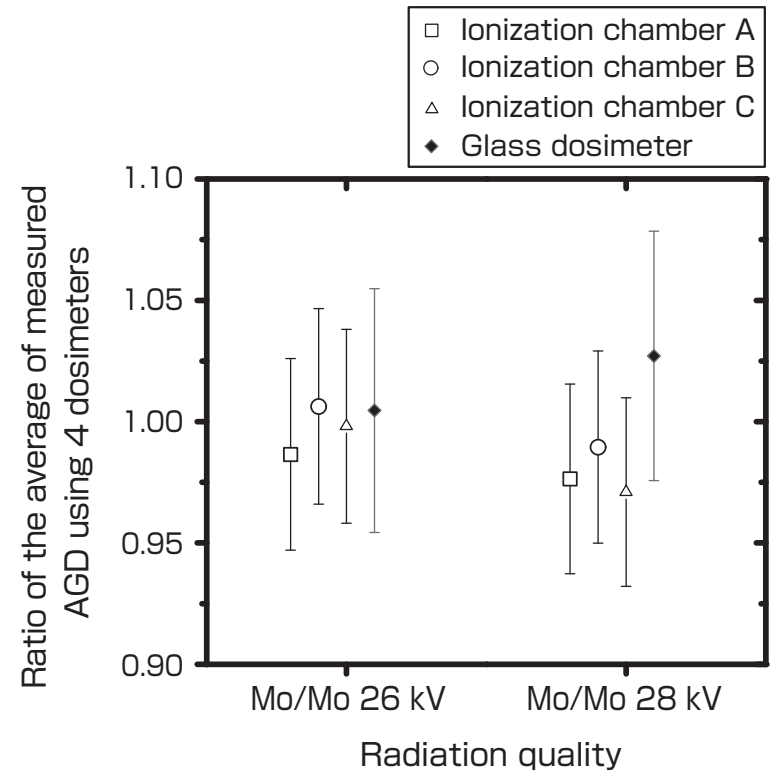

Fig. 10 Comparison of dose measurements for the actual mammography machine

For each data point, the vertical bar shows the uncertainty at the $95 \%$ confidence level. radiation qualities and has established a calibration service to improve the reliability of mammography dose evaluation in medical practice. The time required to develop a mammography dose standard was greatly reduced by utilizing the existing primary standard instrument (free air ionization chamber) for soft X-rays. Additionally, AIST participated in an international key comparison to confirm international equivalence. Rapid and widespread dissemination of the standard was possible by fully utilizing the existing calibration service system. The calibration service system will be further developed in the future through collaborations with academic societies and calibration services.

Currently, digital mammography, which uses digital images and computers, is becoming common instead of conventional screen-film mammography. Compared to evaluation using a conventional film image, dose evaluation from digital image is thought to be more difficult; therefore, the importance of dosimetry in mammography quality control is expected to increase. Additionally, various radiation qualities (W/Rh or $\mathrm{W} / \mathrm{Ag}$ ) will be used in digital mammography. The sensitivity of the semiconductor dosimeter used in medical practice changes significantly depending on the radiation quality, and the development of a reference field is urgently required. Currently, a reference field for digital mammography is being developed in the US, and focused research is being performed to evaluate the semiconductor dosimeter. AIST will continue developing its reference field to manage the increase of radiation qualities efficiently and to contribute to the quality control and safety of advanced mammography.

\section{Acknowledgements}

The excellent quality control system in Japan played a major role in the rapid and widespread dissemination of this standard. We are deeply grateful to the staff members who constructed such an excellent quality control system. We are also grateful to the people of Chiyoda Technol Corporation who assisted in the development and evaluation of the mammography glass dosimeter.

\section{Terminologiy}

Term 1. Age-adjusted incidence rate: The incidence rate corrected for the age composition of the standard population (1985 population model). This enables comparisons among groups with significantly different age compositions.

Term 2. Age-adjusted mortality rate: The mortality rate corrected for the age composition of the standard population (1985 population model). This enables comparisons among groups with significantly different age compositions.

Term 3. Linear attenuation coefficient: When a single- 
energy photon of strength $I_{0}$ enters and permeates a uniform substance, the strength $I$ of the permeating photon decreases exponentially as the thickness of the material $d(\mathrm{~cm})$ increases, as follows:

$I=I_{0} \times e^{-\mu d}$

The coefficient $\mu\left(\mathrm{cm}^{-1}\right)$ is termed the linear attenuation coefficient.

Term 4. Air kerma: The mean sum of the initial kinetic energies of all the secondary charged particles liberated in the air per unit mass via interaction with uncharged particles. It is expressed in $\mathrm{J} / \mathrm{kg}$ as per the International System of Units; however, the special name, grey (Gy), is also used.

Term 5. Exposure: The absolute value of the mean total charge of the ions of one sign produced when all the electrons and positrons liberated or created by photons incident in the dry air per unit mass are completely stopped in dry air. It is expressed as C/ $\mathrm{kg}$ in the International System of Units.

Term 6. Radiophotoluminescence: The phenomenon in which luminescence occurs in proportion to the dose of radiation that glass is exposed to when ultraviolet rays are irradiated to the luminescence center produced in the glass by irradiation. This phenomenon is exploited in personal dosimeters.

Term 7. Key comparison: Consultative committees from each metrology field established under the International Committee for Weights and Measures (Comité International des Poids et Mesures; CIPM) conduct important international comparisons in their respective fields termed CIPM key comparisons. There are 8 quantities subject to key comparisons in the radiation dose field:

K1: Air kerma for ${ }^{60} \mathrm{Co} \gamma$-rays

K2: Air kerma for low-energy X-rays

K3: Air kerma for medium-energy X-rays

K4: Absorbed dose to water for ${ }^{60} \mathrm{Co} \gamma$-rays

K5: Air kerma for ${ }^{137} \mathrm{Cs} \gamma$-rays

K6: Absorbed dose to water for high energy photons K7: Air kerma for mammography beams

K8: Air kerma rate for high-dose-rate ${ }^{192}$ Ir brachytherapy sources

Other than these 8 quantities, the amount of personal dose, $\beta$-ray absorbed dose, and some others are the subjects of supplementary comparison.

\section{References}

[1] Center for Cancer Control and Information Services, National Cancer Center, Japan. http://ganjoho.jp/public/ statistics/pub/statistics02.html\#prg3_1 (in Japanese).

[2] WHO Mortality Database [database online]. http://www. who.int/healthinfo/morttables/en/

[3] Director, Division of the Health for the Elderly, Health and Welfare Bureau for the Elderly, Ministry of Health, Labour and Welfare: "Gan Yobo Juten Kenko Kyoiku Oyobi GanKenshin No Tameno Shishin” No Ichibu Kaisei Ni Tsuite (On the Partial Amendment to the "Guideline for Focused Health Education for Cancer Prevention and Cancer Screening"), Notification \#65 of the Health and Medical Service Law for the Elderly, March 2000 (in Japanese).

[4] Director, Division of the Health for the Elderly, Health and Welfare Bureau for the Elderly, Ministry of Health, Labour and Welfare: "Gan Yobo Juten Kenko Kyoiku Oyobi GanKenshin No Tameno Shishin” No Ichibu Kaitei Ni Tsuite (On the Partial Amendment to the "Guideline for Focused Health Education for Cancer Prevention and Cancer Screening"), Notification No. 0427001 of the Division of the Health for the Elderly, Health and Welfare Bureau for the Elderly, April 2004 (in Japanese).

[5] Chiiki Hoken Kenko Zoshin Jigyo Hokoku (Report on Regional Public Health Services and Health Promotion Services) (Health Statistics Office, Vital, Health and Social Statistics Division, Ministry of Health Labour and Welfare) http://www.e-stat.go.jp/SG1/estat/eStatTopPortal.do (in Japanese)

[6] Mammography Guideline Diffusion Team, Radiography Section, Japanese Society of Radiological Technology: Radiological Technology Library (14-3) Nyubo Satsuei Seido Kanri Manuaru Kaiteiban (Mammography Quality Control Manual, Revised Edition), Japanese Society of Radiological Technology (2004) (in Japanese).

[7] The Central Committee on Quality Control of Mammographic Screening NPO (ed): Dejitaru Mamogurafi Seido Kanri Manuaru (Manual of Digital Mammography Quality Control Management), Igaku Shoin (2009) (in Japanese).

[8] Mammography Quality Standard Act.

[9] Committee on Quality Assurance in Mammography, American College of Radiology: Mammography Quality Control Manual 1999, American College of Radiology, USA (1999).

[10] European Commission: European Guidelines for Quality Assurance in Breast Cancer Screening and Diagnosis, Fourth Edition, European Communities, Luxembourg (2006).

[11] J. Witzani, H. Bjerke, F. Bochud, I. Csete, M. Denoziere, W. de Vries, K. Ennow, J. E. Grindborg, C. Hourdakis, A. Kosunen, H. M. Kramer, F. Pernicka and T. Sander: Calibration of dosemeters used in mammography with different $\mathrm{x}$ ray qualities: EUROMET Project No. 526, Radiation Protection Dosimetry, 108, 33-45 (2004).

[12] C. Kessler, P. Roger and D. T. Burns: Establishment of reference radiation qualities for mammography, Rapport BIPM 2010/01 (2010).

[13] IEC61267 Ed. 2.0, Medical Diagnostic X-ray Equipment - Radiation Conditions for Use in the Determination of Characteristics, IEC (2005).

[14] T. Tanaka, T. Kurosawa, N. Nouda, T. Matsumoto, N. Saito, S. Matsumoto and K. Fukuda: Reference X ray field for mammography dosimetry in Japan, Proceedings of the International Symposium, Standards, Applications and Quality Assurance in Medical Radiation Dosimetry, 2, 4351 (2011).

[15] C. Kessler, D. T. Burns, T. Tanaka, T. Kurosawa and N. Saito: Key comparison BIPM.RI(I)-K7 of the airkerma standards of the NMIJ, Japan and the BIPM in mammography X-rays, Metrologia, 47, 06024 (2010).

[16] D. T. Burns, A. Nohtomi, N. Saito, T. Kurosawa and N. Takata: Key comparison BIPM.RI(I)-K2 of the air-kerma 
standards of the NMIJ and the BIPM in low-energy x-rays, Metrologia, 45, 06015 (2008).

[17] BIPM Key Comparison Database [database online]. http:// kcdb.bipm.org/

\section{Authors}

\section{Takahiro TANAKA}

Completed the course in Physics at the Graduate School of Science and Technology, Sophia University in 2008. Doctor (Science). Joined AIST in 2008. Researcher at the Ionizing Radiation Section, Quantum Radiation Division, National Metrology Institute of Japan, AIST. Engages in research for the dose standard of soft X-ray and mammography

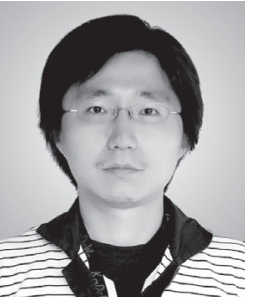
$\mathrm{X}$-ray. In this paper, was mainly in charge of the R\&D and calibration service for the overall mammography dose standard, international key comparison, and drafting of the paper.

\section{Tadahiro KuROSAWA}

Completed the course at the Department of Quantum Science and Energy Engineering, Graduate School of Engineering, Tohoku University in 2000. Doctor (Engineering). Joined the Electrotechnical Laboratory, Agency of Industrial Science and Technology (currently AIST) in 2000. Worked on the $\beta$-ray standard as a visiting researcher

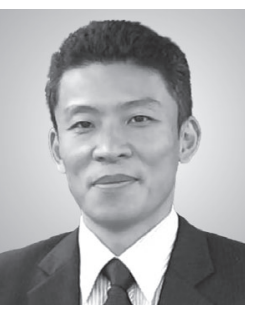
at the Physikalisch-Technische Bundesanstalt (PTB), Germany for three months in 2003. Senior researcher at the Ionizing Radiation Section, Quantum Radiation Division, National Metrology Institute of Japan, AIST. Engages in the development of $\gamma$-ray and X-ray standards. In this paper, was in charge of the Monte Carlo calculation and the international comparison of the mammography dose standard.

\section{Norio SAITo}

Completed the course in Electric Engineering at the Graduate School of Science and Engineering, Waseda University in 1984 . Joined the Electrotechnical Laboratory, Agency of Industrial Science and Technology (currently AIST) in 1984. Doctor (Science). Senior researcher in 1991; senior research at the Ionizing Radiation

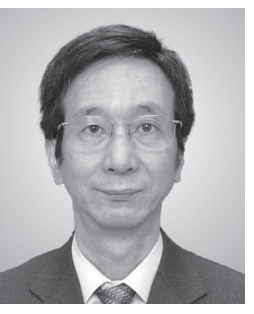
Section, Quantum Radiation Division, National Metrology Institute of Japan, AIST in 2001; section chief in 2005; and division head in 2011. Researcher at the Fritz-Haber-Institut, Germany in 1993 1994. Engages in research of dosimetry standards and radiometry. In this paper, was in charge of planning and organizing the overall concept of mammography dose standard.

\section{Discussions with Reviewers}

\section{Overall evaluation \\ Comment (Naoto Kobayashi, Center for Research Strategy, Waseda University)}

I think this paper is highly significant from the perspective of "establishing the dose standard and traceability for mammography X-rays." Particularly, the major result is that you have achieved this in a short time of about one and half years, from the start of development to the start of dissemination.

\section{Comment (Akira Ono, AIST)}

This paper describes an excellent research result where you set up a solid scenario covering the whole from the establishment of the primary standard for X-ray dose to the development of the standard dissemination system. You realized both the reliability and safety of mammography, and achieved it in a short time. I think there are many points that will be useful to the readers. Also, the firm frame and development of logic as seen in the individual chapters enhance the reliability of the contents and allow easy understanding by the readers.

\section{Utilization of existing resources Comment (Akira Ono)}

In Chapter 1 Introduction you state that the development and dissemination of standards were achieved in a short period of about one and half years "by fully utilizing the existing research facilities and technologies." Also you state that you worked "to construct quick and wide dissemination by incorporating the standards into the current mammography quality control system that existed in private sectors." I think your efforts in utilizing the existing resources and current systems were the keys in conducting the R\&D at a faster pace than usual.

If there are any points that the authors paid attention in utilizing the existing resources and incorporating the standards into current quality control system, please mention them, as they will be useful to other researchers. They may be lessons learned by the authors from this experience.

\section{Answer (Takahiro Tanaka)}

The points to keep in mind when utilizing the existing resources were to note their potential as well as their limitations. For example, the ability to conclude whether the free air ionization chamber, which was the resource used in this research, was capable of sufficient performance as a national standard for mammography radiation quality or not, was required of the researchers. I think the ability to see this affects the success or failure of Full Research.

Additionally, when developing a standard dissemination service, I think it is important to comprehensively understand the process of dose evaluation in the industry and in the academic societies. I think that AIST, the industry, and academic societies had a strong desire to incorporate traceability, the final objective, into the current control system.

\section{Technological issues that were overcome Question (Naoto Kobayashi)}

I ask about the technological issues in achieving the objective of this research. The mammography X-rays are characterized by the low energy of about $30 \mathrm{keV}$. I think the technical point is to use the method of determining the correction factors for the current free air ionization chamber to establish the dose standard. What were the difficult issues that you had to overcome?

Also, there are large differences in the calibration coefficients of the ionization chambers A, B, and C (see Fig. 3). What are the reasons for these differences?

Answer (Takahiro Tanaka)

Although there were several technological issues in the 
development of this standard, there were no major problems. We determined that the issues could be solved by applying the dosimetry technology for low-energy X-rays that had been previously developed at AIST. Specifically, for the primary standard, the dosimetry technology for existing soft X-rays (W/ A1 radiation quality) was used, and correction factors for the primary standard were newly evaluated using the radiation quality of mammography X-rays. Using the Monte Carlo calculation, we confirmed that there would be no major negative effects on the measurement results or uncertainties in the early stages of development. Therefore, we employed this method for standard development.

Other than the above method, there are other ways of developing a new primary standard specifically for mammography $\mathrm{X}$-rays. For example, a free air ionization chamber that is optimized (with small corrections) for mammography radiation quality can be developed. This is a method wherein the correction factors are calculated using the Monte Carlo method (or other methods) and are fed back to the design of the free air ionization chamber for optimal results. BIPM developed the dose standard for mammography using this method. Of course, the uncertainty will be smaller if an optimized primary instrument is developed (at the $95 \%$ confidence level, the uncertainty is $0.6 \%$ for AIST and $0.4 \%$ for BIPM). However, the development period will be longer. While this is an extreme case, BIPM started development in 2001 and embarked on an international key comparison in 2009. In cases where it is necessary to quickly meet the social demand, as in our case, the greatest merit is the shortest possible development period.

Another technological issue was the improvement in the reliability of mammography machine dose evaluation in medical practice. Because the irradiation geometries are different between the reference field of AIST, overseas metrology institutes, and the actual mammography machine, the uncertainty of dose evaluation in medical practice becomes higher. Because mammography $\mathrm{X}$-rays are of low energy, differences in irradiation geometries (e.g., the irradiation distance and presence of compression plates) greatly affect the uncertainty of dose evaluation in addition to the difference in radiation quality. Therefore, we developed a dose standard in which the irradiation geometry was similar to that of a mammography machine, and we tried to reduce the uncertainty in dose evaluation in medical practice. The irradiation distance and compression plate are taken into account by AIST, although they are not considered in overseas standards. However, we were careful to maintain the international compatibility of the standard, and at the same time, we ensured that the radiation quality complied with the IEC standard.

The differences between the calibration coefficients of dosimeters A, B, and C (Fig. 3) may be mainly attributable to differences in the X-ray entrance window material in the ionization chambers as well as the internal structures of the chambers. With low-energy X-rays, a large difference is noted in the energy dependency of the calibration coefficient-even in the ionization chamber dosimeter, which is known to be highly precise. I have added an explanation in the paper.

\section{New efforts to establish a standard dissemination system \\ Question (Naoto Kobayashi)}

On the calibration service system, you write that the standard was disseminated smoothly by having the calibration laboratories bring the client's dosimeter to AIST to do the calibration (subcontracted irradiation test), without fabricating a new calibration facility. You give the example of the glass dosimeter. Were there any new efforts and attempts unseen before connected to this? It seems that it was routine and there was hardly any problem, but how was it actually?

\section{Answer (Takahiro Tanaka)}

The most notable feature of this study was that reliability improvement was performed via evaluation of the glass dosimeter, which is conventionally used in medical practice, in addition to the conventional calibration service system through the calibration of dosimeters.

In the initial plan for standard development, we were thinking about the conventional traceability mediated by dosimeter calibration. I was thinking of the following flow: 1) the dosimeter owned by the calibration laboratory is calibrated at AIST and 2) the calibrated dosimeter is used as a secondary standard to calibrate the user's dosimeter at the X-ray reference field of the calibration laboratory. However, because the radiation quality of mammography X-rays is different from that of the X-rays used in calibration, step 1 with an X-ray field of mammography radiation quality is insufficient, and an X-ray field of mammography radiation quality is needed for step 2 . However, even if there are demands for the standard, the calibration laboratories were of the opinion that the facility investment required to introduce an $\mathrm{X}$-ray irradiation machine would be too expensive. Therefore, we devised a way to disseminate the standard smoothly by having the calibration laboratories use the irradiation facility at AIST.

Although it is estimated that there are approximately 1000 mammography dosimeters distributed throughout Japan, there are only a few calibration laboratories, and I thought that further planning would be needed for widespread standard dissemination. Therefore, in the development phase of this standard, we began looking at the mammography glass dosimeter that was being widely used for dose evaluation in medical practice. By evaluating glass dosimeters according to AIST's dose standard, the reliability of the dose evaluation for many mammography machines will improve. However, there is one problem with evaluating glass dosimeters using this standard: a special reader is needed to read the accumulated dose information from the glass dosimeters. Thus, the dose cannot be determined immediately upon irradiation. Because the irradiation of glass dosimeters at the reference field of AIST and the reading and analysis of the irradiation data were separated, time was required to overcome issues such as the evaluation of uncertainty. Our mammography glass dosimeter is a unique Japanese dosimeter, and I think it has excellent potential.

\section{A comparison of situations in other countries Comment (Naoto Kobayashi)}

In this international key comparison, good results have been obtained as shown in Fig. 9. AIST was the first to participate and obtained good results which show that the international equivalency of the national standard was verified, and this is extremely significant. I think the high quality of AIST will become clearer if you discuss the situation of other countries that participated in the comparison (such as the type and performance of the detectors).

\section{Answer (Takahiro Tanaka)}

In this international key comparison, the transfer ionization chamber was employed by all institutes including AIST. AIST was original in its choice of the transfer standard. Institutes other than AIST selected only 1 transfer standard with a flat energy characteristic in the mammography X-ray energy range. AIST selected 3 types of transfer ionization chambers with different energy dependences to conduct a thorough comparison. As a result, sufficient compatibility was obtained with the BIPM values for all 3 dosimeters. 


\section{Outcome of this research}

\section{Question (Naoto Kobayashi)}

I will ask about the outcome of this research. Now that the standard for low-energy X-ray dose has been newly established and is being disseminated, what practical outcome do you expect? For example, do you expect that the increased reliability of mammography will make more people go to cancer screening? If that will not happen, what other efforts are needed for the further diffusion of mammography in society?

Answer (Takahiro Tanaka)

The reliability of mammography dose evaluation has increased owing to the development of this standard. The manufacturers of mammography machines are making efforts to develop machines with lower dosages and higher quality images. While this standard may not contribute to the improvement of image quality, I think it will contribute to a lower dose, because a dose standard is essential for quantitative dose evaluation.

We have received several offers for joint research from the industry, universities, and academic societies, and we are currently working to increase the precision of mammography dose evaluation. In the future, I hope we can help to reduce the mammography dose using data obtained from joint research projects.

\section{Research goals}

\section{Comment (Naoto Kobayashi)}

Initially, the diagram for the scenario in Fig. 4 showed only the establishment of the standard for general X-ray dosimetry and precision improvement. I don't think it shows the scenario for the establishment and distribution of the mammography X-ray dose evaluation method that you described in this paper.

I think there are the following characteristics in the mammography X-ray dose evaluation. Please add how they were positioned in the scenario, and present the diagram of the scenario for achieving your goal.

(1) The existing soft X-ray standard (W/Al radiation quality) was used as the national standard, and the correction factors of the national standard were newly evaluated for the mammography $\mathrm{X}$-ray radiation quality.

(2) Although there is no technological novelty in (1), it was preliminarily confirmed using the Monte Carlo calculation that there would be no fatal effect on the measurement results and uncertainty.

(3) Rather than developing a primary standard optimized for mammography radiation quality and aiming at high precision, importance was placed on the speed of standard establishment using the existing standard, even if precision was somewhat sacrificed. (This is an extremely important R\&D strategy.)
(4) The objective was set as the improvement of the reliability of the dose evaluation of mammography machines in medical practice. Therefore, development effort was made to create a dose standard with irradiation geometry close to the mammography machine, aiming for the reduction of the uncertainty of dose evaluation in medical practice.

(5) Moreover, in standard dissemination, focus was placed on the glass dosimeter used in medical practice. Attempt was made to improve the reliability of dose evaluation of mammography machines by evaluating the glass dosimeters using AIST's dose standard.

\section{Answer (Takahiro Tanaka)}

I revised the scenario diagram as you have indicated to clarify the characteristics of this research. Additionally, I have revised the arrangement of the chapters following chapter 4 to match the scenario diagram.

\section{Technological characteristics of the standard in this research Question (Naoto Kobayashi)}

I ask the following questions for confirmation. Are the following reasons valid in terms of the difference from the conventional X-ray dose standard?

(1) It is necessary to use low energy of about $30 \mathrm{keV}$ instead of $80 \mathrm{keV}$ to reduce the effect of radiation to the human body. Also, since the absorption per unit length (stopping power) of the X-rays within that energy range is greater than that of the medium energy X-rays, sufficient contrast can be obtained between the mammary tissues and the lesions. On the other hand, special measures must be taken because the precision of $\mathrm{X}$-ray dose evaluation decreases for low energy X-rays.

(2) Since an X-ray tube with a molybdenum target anode and a molybdenum filter are used for the mammography X-ray beam at around $30 \mathrm{keV}$, the energy spectrum approaches monochromatic, and the energy spectrum after absorption by substances is greatly different from the X-rays that use W/Al at around $80 \mathrm{keV}$.

\section{Answer (Takahiro Tanaka)}

I think your understanding is correct. To obtain sufficient contrast between the mammary tissues and lesions, an energy lower than that used in general radiography is needed for mammography. In the dosimetry of low-energy X-rays where the sensitivity of the dosimeter changes with the X-ray energy, the difference between the radiation qualities of general radiography $\mathrm{X}$-rays and mammography X-rays affects the precision of the dose evaluation. The paper was revised to address the radiation quality of mammography X-rays. 